\title{
Editorial
}

\section{Depression in diabetes: The need to screen}

\author{
Yatan Pal Singh Balhara, Sanjay Kalra ${ }^{1}$, Ashok K Das ${ }^{2}$ \\ National Drug Dependence Treatment Centre, All India Institute of Medical Sciences, New Delhi, ${ }^{1}$ Department of Endocrinology, Bharti Hospital and \\ B.R.I.D.E., Karnal, Haryana, ${ }^{2}$ Department of Medicine, Jawaharlal Institute of Post Graduate Medical Education and Research, Puducherry, India
}

Most diabetes care professionals are aware of the 'Rule of Halves', which states that only half of all people with diabetes are diagnosed, half of those diagnosed receive treatment, and half of those who do get treatment reach therapeutic targets. ${ }^{[1]}$ A similar rule prevails in depression as well. It is estimated that $12.5 \%$ of all patients in primary care have experienced major depressive disorder in the past year of these, only half are recognized clinically, of which only another half receive treatment, of those who do receive treatment, only $40 \%$ are treated adequately, and of these two-thirds achieve remission. Finally, only six in 100 persons with depression achieve optimal therapeutic targets. $^{[2]}$

That depression and diabetes are interlinked with each other is an indisputable fact. Indian journals, including the Journal of Social Health in Diabetes, have carried reviews and original articles highlighting this aspect of endocrinology. ${ }^{[3,4]}$ The bidirectional relationship of diabetes and depression has also been explored in these pages. This means that just as diabetes is more common in persons with depression, depression is more prevalent in individuals with diabetes. A corollary to this is that just as people with depression should be assessed for diabetes; those with diabetes must be screened for depression.

From a diabetologist's viewpoint, this is easier said than done. Diagnosis for mental health disorders conventionally involves use of lengthy interviews and/or multi-item questionnaires, which put a significant strain on already

\begin{tabular}{|l|l|}
\hline \multicolumn{2}{|c|}{ Access this article online } \\
\hline Quick Response Code: & Website: \\
\hline & www.joshd.net \\
\hline & \\
\hline
\end{tabular}

limited resource of time in clinical settings. Self-reported questionnaires bring with them the pre-requisite of adequate literacy levels, while physician-rated questionnaires are too labor-intensive for routine use.

Diabetologists, who are usually trained in the biomedical approach to health, may find it challenging to appreciate the importance of the psychosocial components nested in the bio-psycho-social model of disease. While some may understand the importance of psychosocial factors in the natural history of diabetes, they lack the confidence and expertise to assess them. Yet others may avoid screening for mental health disorders, as they feel there is no credible intervention to provide if these conditions are detected.

A theoretical (and often ill-founded) objection to the concept of routine screening for depression in a diabetes care clinic is the 'nocebo' effect-the fear that a healthy person may develop depressive symptoms subsequent to a screening procedure. Finally, some physicians may rely heavily on their gut feeling thinking they will be able to sense it when it appears and consequently forgo a systematic approach to detection of depression among their patients.

Measurement is an essential part of modern medical praxis. Required for baseline assessment, periodic monitoring, and evaluation of therapeutic strategies, and interventions; no outcome can be defined objectively and meaningfully without measurement. Clinicians in all specialties, including diabetology, are familiar with quantitative assessment of biological markers of disease, such as glucose, cholesterol, and blood pressure.

All these variables are checked by validated, quality controlled, instruments which provide replicable results. The same principles are applicable to the practice of psychiatry. Validated and replicable instruments are used for case-finding and monitoring purposes. Depression

Corresponding Author: Dr. Yatan Pal Singh Balhara, National Drug Dependence Treatment Centre, All India Institute of Medical Sciences, New Delhi - 110 029, India. E-mail: ypsbalhara@gmail.com 
screening, for example, can be done using Patient Health Questionnaire-9, ${ }^{[5]}$ Patient Health Questionnaire-2, ${ }^{[6]}$ and two-item case-finding instrument. ${ }^{[7]}$

It is worth making a specific reference to the two-item case finding instrument proposed by Whooley et al., ${ }^{[7]}$ while being brief and simple to administer it has been found to have acceptable sensitivity and specificity of $96 \%$ and $57 \%$, respectively, in identifying depression. It includes two questions taken from the 27-item Primary Care Evaluation of Mental Disorders Procedure PRIME-MD. ${ }^{[5]}$ These two questions focus on altered mood and anhedonia-two cardinal symptoms of depression.

These two questions are:

1. During the past month, have you often been bothered by feeling down, depressed, or hopeless?

2. During the past month, have you often been bothered by little interest or pleasure in doing things?

An answer of yes to either of these two questions warrants a detailed evaluation for depressive disorder. The Whooley questions can be utilized as a simple, time-saving, validated instrument to screen for depression in diabetes care centers.

Another short, easy-to-administer tool is the five-item World Health Organization Well Being Index that assesses the psychological well-being. ${ }^{[8]}$ The subjective quality of life belongs to the dimension of psychological well-being such as positive mood, vitality, and interest in things. The WHO-Five well-being index has been derived from a larger rating scale developed for a WHO project on quality of life in patients suffering from diabetes. It includes five items that assess positive mood (good spirits, relaxation), vitality (being active and waking up fresh and rested), and general interests (being interested in things). Each of the five items is rated on a 6-point Likert scale from 0 (=not present) to 5 (constantly present). A raw score less than 13 or an answer of 0 to 1 to any of the five items warrants detailed evaluation for depressive disorder.

Free to use, with no hassles of copyright, non-time consuming and easy to understand-these instruments are extremely user-friendly. While these short, simple, questionnaires do not have the high specificity of other case-finding instruments, their high sensitivity makes them suitable for use as screening tools.
As we strive to enhance our understanding of diabetes as a bio-psycho-social disorder, it is imperative to focus on the psychosocial concomitants of the condition. For a clinical perspective such an exercise will be most fruitful if one can minimize the inadvertent delay in identifying these issues. Diabetes care professionals should incorporate the use of easy case-finding instruments, such as Whooley's questions and the WHO (Five) Well-Being Index in their routine practice. Professional organizations across the globe should follow the example set by national guidelines in India, Germany, and other countries, which recommend regular assessment of psychological well being as an integral part of diabetes care. ${ }^{[9]}$

The Journal of Social Health in Diabetes hopes to contribute to this awareness and improved clinical practices in diabetes clinics across the world, by calling for routine screening of depression in diabetes using validated case-finding instruments.

\section{REFERENCES}

1. Hart JT. Rule of halves: Implications of increasing diagnosis and reducing dropout for future workload and prescribing costs in primary care. Br J Gen Pract 1992;42:116-9.

2. Pence BW, O'Donnell JK, Gaynes BN. The depression treatment cascade in primary care: A public health perspective. Curr Psychiatry Rep 2012;14:328-35.

3. Balhara YP. Diabetes and psychiatric disorders. Indian J Endocrinol Metab 2011;15:274-83.

4. Balhara YP, Verma R. Management of depression in diabetes: A review of psycho-social interventions. J Soc Health Diabetes 2013;1:22-6.

5. Spitzer RL, Kroenke K, Williams JB. Validation and utility of a self-report version of PRIME-MD: The PHQ primary care study. Primary Care Evaluation of Mental Disorders. Patient Health Questionnaire. JAMA 1999;282:1737-44.

6. Richardson LP, Rockhill C, Russo JE, Grossman DC, Richards J, McCarty C, et al. Evaluation of the PHQ-2 as a brief screen for detecting major depression among adolescents. Pediatrics 2010;125:e1097-103.

7. Whooley MA, Avins AL, Miranda J, Browner WS. Case-finding instruments for depression. Two questions are as good as many. J Gen Intern Med 1997;12:439-45.

8. WHO. (Five) Well-Being Index (1998 version): Available from: http://cure4you.dk/354/WHO-5_English.pdf [Last accessed on 2013 Mar 22].

9. Kalra S, Sridhar G, Balhara YP, Sahay R, Bantwal G, Baruah M, et al. National recommendations: Psychosocial management of diabetes in India. Indian J Endocrinol Metab 2013;17:376-95.

How to cite this article: Balhara YS, Kalra S, Das AK. Depression in diabetes: The need to screen. J Soc Health Diabetes 2013;1:51-2.

Source of Support: Nil. Conflict of Interest: None declared. 